\title{
Conversion and conservation of light energy in a photosynthetic microbial mat ecosystem
}

\author{
Mohammad AA Al-Najjar ${ }^{1}$, Dirk de Beer ${ }^{1}$, Bo Barker Jørgensen ${ }^{1}$, Michael Kühl ${ }^{2}$ \\ and Lubos Polerecky ${ }^{1}$ \\ ${ }^{1}$ Max-Planck Institute for Marine Microbiology, Celsiusstrasse, Bremen, Germany and ${ }^{2}$ Marine Biological \\ Laboratory, Department of Biology, University of Copenhagen, Strandpromenaden, Helsingør, Denmark
}

\begin{abstract}
Here we present, to the best of our knowledge, the first balanced light energy budget for a benthic microbial mat ecosystem, and show how the budget and the spatial distribution of the local photosynthetic efficiencies within the euphotic zone depend on the absorbed irradiance $\left(J_{\text {abs }}\right)$. Our approach uses microscale measurements of the rates of heat dissipation, gross photosynthesis and light absorption in the system, and a model describing light propagation and conversion in a scattering-absorbing medium. The energy budget was dominated by heat dissipation on the expense of photosynthesis: in light-limiting conditions, $95.5 \%$ of the absorbed light energy dissipated as heat and $4.5 \%$ was channeled into photosynthesis. This energy disproportionation changed in favor of heat dissipation at increasing irradiance, with $>99 \%$ of the absorbed light energy being dissipated as heat and $<1 \%$ used by photosynthesis at $J_{\text {abs }}>700 \mu$ mol photon $\mathrm{m}^{-2} \mathrm{~s}^{-1}\left(>150 \mathrm{~J} \mathrm{~m}^{-2} \mathrm{~s}^{-1}\right)$. Maximum photosynthetic efficiencies varied with depth in the euphotic zone between $0.014-0.047 \mathrm{O}_{2}$ per photon. Owing to steep light gradients, photosynthetic efficiencies varied differently with increasing

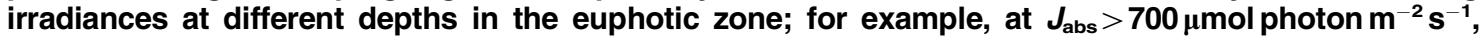
they reached around $10 \%$ of the maximum values at depths $0-0.3 \mathrm{~mm}$ and progressively increased toward $100 \%$ below $0.3 \mathrm{~mm}$. This study provides the base for addressing, in much more detail, the photobiology of densely populated photosynthetic systems with intense absorption and scattering. Furthermore, our analysis has promising applications in other areas of photosynthesis research, such as plant biology and biotechnology.
\end{abstract}

The ISME Journal (2010) 4, 440-449; doi:10.1038/ismej.2009.121; published online 12 November 2009

Subject Category: microbial ecosystem impacts

Keywords: efficiency; light conversion; microbial mats; microsensors; photosynthesis

\section{Introduction}

About $3.8 \times 10^{24} \mathrm{~J}$ of solar energy is anually absorbed by the Earth's surface and atmosphere. The total energy consumption of humans in the year 2007 was $0.01 \%$ of this flux, whereas the primary productivity of global ecosystems is estimated at around $0.1 \%$ (Makarieva et al., 2008). Thus, the amount of solar energy that is available but not yet harvested is enormous. Photosynthesis offers a possibility to use this energy source, as it is a mechanism by which solar energy is converted into chemical energy and stored as biomass in phototrophic organisms, such as plants, algae or cyanobacteria. Once stored, the organic material can serve as food for heterotrophic organisms, or can be further converted to other forms of usable energy such as fuel (Ragauskas et al., 2006). Therefore, the study of photosynthetic

Correspondence: M Al-Najjar, Microsensor Research Group, Max-Planck institute for marine microbiology, Celsiusstrasse 1, Bremen 28359, Germany.

E-mail: malnajja@mpi-bremen.de

Received 11 August 2009; revised 5 October 2009; accepted 8 October 2009; published online 12 November 2009 efficiency is a very active research field, especially in recent years because of a growing demand for biofuels and technologies for sustainable energy production (Ragauskas et al., 2006; Mussgnug et al., 2007; Dismukes et al., 2008; Rosenberg et al., 2008).

Research on photosynthetic efficiency has mainly focused on plants (Singsaas et al., 2001; Zhu et al., 2008) and planktonic algae (Dubinsky et al., 1986; Flameling and Kromkamp, 1998; Rosenberg et al., 2008). The measurements are typically conducted in a homogeneous light field allowing a uniform transfer of light energy to the studied photosynthetic system, and thus facilitating detailed studies of underlying physiological mechanisms of light adaptation and acclimation. Benthic photosynthetic systems, such as biofilms and microbial mats, constitute an important component of shallow water habitats by contributing significantly to primary productivity (Cahoon, 1999; Guerrero et al., 2002), but the photosynthetic efficiency and energy budget for such systems are virtually unexplored. Photosynthetic microbial mats are complex and highly compacted microbial ecosystems, where a high diversity of phototrophic and heterotrophic populations interact within a few millimeters thick 
photosynthetically active (euphotic) zone (van Gemerden, 1993; Stal, 2000). Owing to the high concentration of cells and the presence of abiotic components, such as sediment particles, these benthic systems are fundamentally different from the terrestrial and planktonic systems investigated so far in that the light field is highly heterogeneous on spatial scales comparable with the size of and the distance between the individual organisms (Kühl and Jørgensen, 1992, 1994; Kühl et al., 1994). To what extent such pronounced light gradients affect the photosynthetic efficiency of the individual cells and the energy budget of these microbial ecosystems as a whole is still an open question.

In photosynthesis research, the quantum efficiency (QE) is a frequently used measure of light conversion, as the photochemical reactions are driven by photons that are absorbed by the chlorophylls and their antenna in the photosystems, and lead to a charge separation across the thylakoid membrane. The photosynthetic QE is defined as the amount of $\mathrm{CO}_{2}$ molecules assimilated per number of photons absorbed. Assuming a 1:1 stoichiometry of $\mathrm{CO}_{2}$ fixation and $\mathrm{O}_{2}$ production, which is reasonable when $\mathrm{NH}_{4}^{+}$is the predominant source of nitrogen, rather than $\mathrm{NO}_{3}^{-}$, QE can also be determined from the amount of $\mathrm{O}_{2}$ molecules produced per photon absorbed. QE is typically derived either from $\mathrm{CO}_{2}$ or $\mathrm{O}_{2}$ exchange measurements performed in flux chambers or by fluorimetry using variable chlorophyll $a$ fluorescence measuring the quantum efficiency of photosystem II (Falkowski and Raven, 1997). Assuming no losses, the maximal QE of photosynthesis is 0.125 . This follows from the basic photon requirements for the amount of electrons that need to be transferred to oxidize water and reduce $\mathrm{CO}_{2}$, and from the fact that this electron transfer takes place sequentially over two reaction centers, each requiring one photon to separate one electron (thus, requiring in total eight photons per $\mathrm{CO}_{2}$ molecule fixed or per $\mathrm{O}_{2}$ molecule produced). Under light-limiting conditions, QE in terrestrial plants and planktonic algae can reach up to 0.1100.111 (see Supplementary Table S1), which is indeed close to the theoretical maximum.

The energy efficiency (EE) is the preferred measure when considering photosynthesis in the context of energy production. According to definition, EE is the ratio between the energy stored as biomass and the absorbed light energy, and can be quantified directly from biomass growth experiments. Alternatively, EE can be derived from QE by considering the following steps: first, in the lightdependent reactions, in which $\mathrm{O}_{2}$ is formed by splitting of water, reducing equivalents are used to form nicotinamide adenine dinucleotide phosphate (NADPH), and ATP is formed by the proton motive force, the energy gained and stored is $E_{\mathrm{G}}=482.9 \mathrm{~kJ}$ $\left(\mathrm{mol} \mathrm{O}_{2}\right)^{-1}$. This follows from the Gibbs free energies of reactants in the reactions $2 \mathrm{H}_{2} \mathrm{O}+$ $2 \mathrm{NADP}^{+} \rightarrow \mathrm{O}_{2}+2 \mathrm{NADPH}+2 \mathrm{H}^{+}\left(\Delta G=439 \mathrm{~kJ} \mathrm{~mol}^{-1}\right)$ and $\mathrm{ADP}+\mathrm{P}_{i} \rightarrow \operatorname{ATP}\left(\Delta G=43.9 \mathrm{~kJ} \mathrm{~mol}^{-1}\right.$, both at $\mathrm{pH} 7$; Thauer et al., 1977). Second, the molar energy content of light with wavelength $\lambda$ is $E_{\lambda}=N_{\mathrm{A}}(h c / \lambda)$, where $N_{\mathrm{A}}, h$ and $c$ are the Avogadro's number, the Planck constant and the speed of light, respectively. Thus, assuming no intermediate losses, that is, the energy stored in the light-dependent reaction is fully used for $\mathrm{CO}_{2}$ fixation in the dark reaction (equivalent to the assumption of $\mathrm{O}_{2}: \mathrm{CO}_{2}$ stoichiometry being equal to 1), a relationship between $\mathrm{EE}$ and $\mathrm{QE}$ can be written as

$$
\mathrm{EE}=\frac{E_{\mathrm{G}}}{E_{\lambda}} \mathrm{QE}
$$

Considering blue $(450 \mathrm{~nm})$ and red $(670 \mathrm{~nm})$ photons, which are most efficiently absorbed by chlorophyll $a$, the factor $E_{\mathrm{G}} / E_{\lambda}$ ranges between 1.82.7 photon per $\mathrm{O}_{2}$, averaging at 2.22 photon per $\mathrm{O}_{2}$ for an 'average' photon $(550 \mathrm{~nm})$ within the range of photosynthetically active radiation (PAR, 400$700 \mathrm{~nm})$. Thus, the theoretical maximum EE of photosynthetic light utilization is about $27.7 \%$. This represents a situation in which all incident photons are absorbed and used for $\mathrm{O}_{2}$ evolution and equivalent $\mathrm{CO}_{2}$ assimilation. The actual photosynthetic EE is lower, primarily because (i) capture of photons by photopigments and channeling of their energy to the reaction center is inefficient, (ii) photons are absorbed by cell components and accessory pigments that are not photosynthetically active, (iii) excess excitation energy is dissipated as heat by non-photochemical quenching processes, and (iv) part of the energy stored in the lightdependent reaction is used for processes other than $\mathrm{CO}_{2}$ fixation (Schneider, 1973; Osmond, 1994; Huner et al., 1998; Zhu et al., 2008). Direct biomass growth measurements for terrestrial C3 and C4 plants obtained EE values of 4.6 and $6.0 \%$, respectively (Supplementary Table S1; Zhu et al., 2008). So far the photosynthetic EE in benthic biofilms and microbial mats is unexplored.

Detailed studies of microbenthic photosynthesis (PS) require specialized tools and methods to resolve the distribution of light and photosynthetic activity at relevant spatial scales. Oxygen microsensors can resolve the steep concentration gradients of $\mathrm{O}_{2}$ in microbial mats at $\mu \mathrm{m}$ resolution and the light-dependent $\mathrm{O}_{2}$ dynamics at $0.1-0.2 \mathrm{~s}$ resolution (Revsbech et al., 1983; Revsbech and Jørgensen, 1986). Using the so-called light-dark shift method (Revsbech and Jørgensen, 1983), $\mathrm{O}_{2}$ microsensors can measure the volumetric rates of gross photosynthesis with a spatial resolution of $100-200 \mu \mathrm{m}$. When combined with measurements of the locally available light using fiber-optic microprobes (Kühl, 2005), the vertical distribution of the relative photosynthetic efficiency within a microbial mat can be quantified (Lassen et al., 1992b). Microscale measurements of volumetric gross PS are typically done throughout the mm-thin euphotic zone under 
different incident irradiance levels and are then integrated over depth to yield the areal rate of gross PS for the entire ecosystem as a function of the incident irradiance. We compiled such data from a number of sources and, assuming that $90 \%$ of the incident irradiance was absorbed and used, obtained maximal QE for benthic photosynthetic systems in the range of 0.01-0.07 (Supplementary Table S1). In principle, Equation 1 could be used to simply convert QE to EE, but this approach would not satisfactorily address the fundamental question of how the energy budget of a microbial mat ecosystem is balanced between the incident downwelling irradiance, upwelling irradiance, photosynthesis and heat dissipation, especially as a function of depth spanning the steep light and chemical gradients across the euphotic zone.

This study provides, to the best of our knowledge, the first complete energy budget assessment in a cyanobacterial mat, a model representative of a microphytobenthic ecosystem. We aimed to quantify how much of the incident light energy flux $\left(J_{\text {IN }}\right)$ is back-scattered and thus lost to the environment $\left(J_{R}\right)$, dissipated as heat $\left(J_{\mathrm{H}}\right)$ or chemically stored by photosynthesis $\left(J_{\mathrm{PS}}\right)$, and how this energy flow varies with depth across the euphotic zone. This general aim was divided into three main challenges: (i) to experimentally verify that $J_{\mathrm{IN}}=J_{\mathrm{R}}+J_{\mathrm{H}}+J_{\mathrm{PS}}$, (ii) to characterize how the budget depends on the incident irradiance, and (iii) to elucidate how the overall photosynthetic light utilization efficiency of the entire mat depends on the partial efficiencies of the different layers in the mat. We applied microsensors for $\mathrm{O}_{2}$, temperature and scalar irradiance to resolve the vertical variability of the energy conversion in the mat ecosystem, and analysed the data based on a new model that describes light propagation and conversion in a medium with intense absorption and scattering (see Supplementary Information).

\section{Materials and methods}

The studied cyanobacterial mat originated from an intertidal flat near Abu Dhabi (United Arab Emirates), and was kept in the laboratory in $0.2 \mu \mathrm{m}$ filtered seawater (originating from the North Sea) under a 10-h light-14-h dark illumination regime at incident irradiance $\sim 480 \mu \mathrm{mol}$ photon $\mathrm{m}^{-2} \mathrm{~s}^{-1}$ (spectral composition similar to sunlight) before measurements. The measurements were conducted in a thermostated flow chamber that was vertically illuminated with a collimated light beam of PAR from a tungsten halogen lamp. A stable laminar flow of filtered aerated seawater (temperature $23^{\circ} \mathrm{C}$, salinity 35) above the mat surface was maintained throughout the measurements.

High spatial resolution measurements of $\mathrm{O}_{2}$ concentration, temperature and scalar irradiance were performed with a fast-responding Clark-type micro- electrode (tip diameter $\sim 30 \mu \mathrm{m}$; Revsbech, 1989), a thermocouple microsensor (tip diameter $\sim 50 \mu \mathrm{m}$; T50, Unisense A/S, Aarhus, Denmark) and a fiberoptic scalar irradiance microprobe (integrating sphere diameter $\sim 100 \mu \mathrm{m}$; Lassen et al., 1992a) connected to a spectrometer (USB4000, Ocean Optics, Dunedin, FL, USA), respectively. The downwelling spectral quantum irradiance $\left(I_{\lambda}\right.$ in $\mu$ mol photon $\mathrm{m}^{-2} \mathrm{~s}^{-1} \mathrm{~nm}^{-1}$ ) was measured by a spectrometer equipped with a cosine collector and intercalibrated against a PAR quantum irradiance sensor (LI-190 Quantum) connected to a light meter (LI-250, both from LI-COR Biosciences, Lincoln, NE, USA). The spectral irradiance reflectance of the mat $\left(R_{\lambda}\right)$ was calculated as $R_{\lambda}=I_{\lambda \text {,mat }} / I_{\lambda \text {,ref }}$, where $I_{\lambda, \text { mat }}$ and $I_{\lambda, \text { ref }}$ are the back-scattered spectral radiances measured above the mat and above a white reflectance standard (Spectralon; Labsphere, North Sutton, NH, USA), respectively, with a fiberoptic field radiance microprobe (Jørgensen and des Marais, 1988). This calculation assumed that the light back-scattered by the mat was diffused, which is typically the case for highly scattering benthic microbial communities (Kühl and Jørgensen, 1994).

The light energy absorbed in the mat $\left(J_{\text {abs }}\right.$ in $\mathrm{J} \mathrm{m}^{-2} \mathrm{~s}^{-1}$ ) was calculated as the vector irradiance by subtracting the downwelling and upwelling irradiance:

$$
J_{\mathrm{abs}}=\int_{400}^{700} I_{\lambda} E_{\lambda}\left(1-R_{\lambda}\right) \mathrm{d} \lambda
$$

where $E_{\lambda}$ is the energy of a photon with wavelength $\lambda$ (see Introduction).

The incident light energy that was absorbed by the mat but not conserved as chemical energy by photosynthesis resulted in an increase of the mat temperature relative to that of the overlaying water. This allowed calculation of the areal rate of heat dissipation inside the euphotic zone of the mat $\left(J_{\mathrm{H}}\right.$ in $\mathrm{J} \mathrm{m}^{-2} \mathrm{~s}^{-1}$ ) from Fourier's law of conduction, that is,

$$
J_{\mathrm{H}}=k \frac{\partial T}{\partial z},
$$

where $k$ is the thermal conductivity in water $\left(0.6 \mathrm{~J} \mathrm{~m}^{-1} \mathrm{~s}^{-1} \mathrm{~K}^{-1}\right)$ and $\partial T / \partial z$ is the temperature gradient measured in the thermal boundary layer (Jimenez et al., 2008).

Volumetric rates of gross photosynthesis $(P$ in $\mu \mathrm{mol} \mathrm{O} \mathrm{m}^{-3} \mathrm{~s}^{-1}$ ) were measured by the light-dark shift method (Revsbech and Jørgensen, 1983) in vertical steps of $100 \mu \mathrm{m}$ throughout the upper layers of the microbial mat until no photosynthesis was detected. Areal rates of photosynthesis $\left(P_{\mathrm{a}}\right.$ in $\mu \mathrm{mol}$ $\mathrm{O}_{2} \mathrm{~m}^{-2} \mathrm{~s}^{-1}$ ) were calculated by integrating the volumetric rates over the euphotic zone. The flux of energy conserved by photosynthesis $\left(J_{\mathrm{PS}}\right.$ in $\left.\mathrm{Jm}^{-2} \mathrm{~s}^{-1}\right)$ was calculated as $J_{\mathrm{PS}}=E_{\mathrm{G}} P_{\mathrm{a}}$, where $E_{\mathrm{G}}=482.9 \mathrm{~kJ}$ 
$(\mathrm{mol} \mathrm{O})^{-1}$ is the Gibbs energy produced when $\mathrm{O}_{2}$ is formed by splitting water (see Introduction). This expression quantifies the total flux of photosynthetically conserved energy, including all other subsequent energy conversions and mineralization processes taking place in the mat, such as excretion of carbohydrates and their subsequent respiration and/or incorporation into biomass by heterotrophic bacteria.

For a given absorbed light energy, $J_{\text {abs }}$, the efficiencies of photosynthetic energy conservation $\left(\varepsilon_{\mathrm{PS}}\right)$ and heat dissipation $\left(\varepsilon_{\mathrm{H}}\right)$ for the entire euphotic zone of the mat were calculated from the energy fluxes as

$$
\varepsilon_{\mathrm{PS}}=\frac{J_{\mathrm{PS}}\left(J_{\mathrm{abs}}\right)}{J_{\mathrm{abs}}} \text { and } \varepsilon_{\mathrm{H}}=\frac{J_{\mathrm{H}}\left(J_{\mathrm{abs}}\right)}{J_{\mathrm{abs}}} .
$$

In light-limiting conditions, that is, at $J_{\mathrm{abs}} \rightarrow 0$, the efficiencies were estimated as follows: First, the measured $J_{\mathrm{PS}}$ as a function of $J_{\mathrm{abs}}$ was fitted with the saturated exponential model (Webb et al., 1974)

$$
J_{\mathrm{PS}}\left(J_{\mathrm{abs}}\right)=J_{\mathrm{PS}, \max }\left(1-\mathrm{e}^{-J_{\mathrm{abs}} / E_{\mathrm{k}}}\right)
$$

to estimate the maximum photochemically conserved energy flux, $J_{\mathrm{PS}, \max }$, and the parameter $E_{\mathrm{k}}$ characterizing the apparent photochemical light adaptation. Subsequently, it was assumed that autofluorescence from the mat is negligible and thus the energy budget in the mat satisfies the Equation $J_{\mathrm{abs}}=J_{\mathrm{PS}}+J_{\mathrm{H}}$, that is, $\varepsilon_{\mathrm{PS}}+\varepsilon_{\mathrm{H}}=1$. The respective efficiencies in light-limiting conditions where then calculated as

$$
\varepsilon_{\mathrm{PS}, \text { max }}=\frac{J_{\mathrm{PS}, \text { max }}}{E_{\mathrm{k}}} \text { and } \varepsilon_{\mathrm{H}, \text { min }}=1-\varepsilon_{\mathrm{PS}, \text { max }}
$$

The regulation of the photosynthetic efficiency by light in each layer within the euphotic zone was assessed as follows. First, the locally measured scalar irradiance integrated over PAR, $E_{s}$, was fitted with an exponential function $E_{\mathrm{s}}(z)=E_{\mathrm{s}}(0)$ $\exp \left[-\alpha\left(z-z_{0}\right)\right]$ to estimate the light attenuation coefficient, $\alpha$. Then, assuming a totally diffuse light field inside the mat (that is, photons at each depth propagated in all directions with equal probabilities), the local density of absorbed light $\left(\tilde{E}_{\text {abs }}(z)\right.$ in $\mu \mathrm{mol}$ photon $\left.\mathrm{m}^{-3} \mathrm{~s}^{-1}\right)$ was calculated as $\tilde{E}_{\text {abs }}(z)=$ $(\mathrm{K} / 2) E_{\mathrm{s}}(z)$ (see derivation in Supplementary Information), where the absorption coefficient $K$ is related to $\alpha$ and the irradiance reflectance $R$ as

$$
K=\alpha \frac{1-R}{1+R} .
$$

Finally, the local photosynthetic efficiency, $\eta$, was calculated by dividing the locally measured volumetric rates of gross photosynthesis with $\tilde{E}_{\text {abs }}(\mathrm{z})$, that is,

$$
\eta(z)=\frac{P(z)}{\tilde{E}_{\mathrm{abs}}(z)}=\frac{2 P(z)}{K E_{\mathrm{s}}(z)}
$$

Furthermore, the local photosynthesis rates as a function of $\tilde{E}_{\text {abs }}$ were fitted with the saturated exponential model (Equation 5) to yield the maximum photosynhesis rates $P_{\max }(z)$ and parameters $\tilde{E_{\mathrm{k}}}(z)$, from which the maximum local quantum efficiencies were calculated as $\eta_{\max }(z)=P_{\max }(z) / \tilde{E}_{\mathrm{k}}(z)$.

More detailed descriptions of materials, methods and derivations are given as Supplementary Information.

\section{Results}

The energy budget was investigated for incident irradiances spanning a range of 20-1300 $\mu \mathrm{mol}$ photon $\mathrm{m}^{-2} \mathrm{~s}^{-1}$. At all levels, scalar irradiance inside the microbial mat was attenuated exponentially with depth below $0.2 \mathrm{~mm}$, reaching $1 \%$ of the surface level at $\sim 1.2 \mathrm{~mm}$ (Figure 1a). The
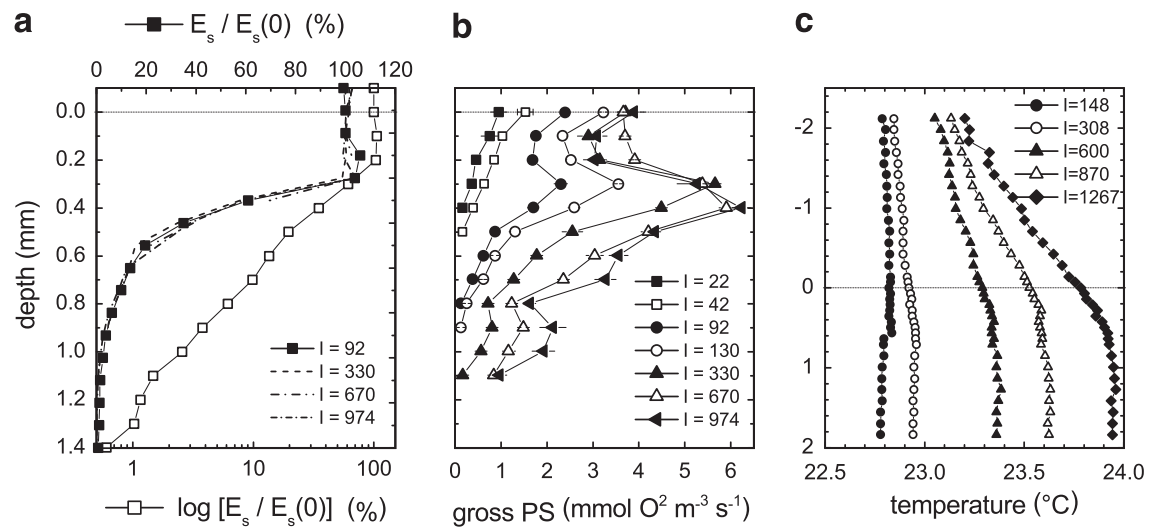

Figure 1 Vertical microprofiles of (a) scalar irradiance (in \% of surface scalar irradiance), (b) volumetric rates of gross photosynthesis, and (c) temperature, measured in the studied microbial mat at increasing incident downwelling irradiances (legend in $\mu \mathrm{mol}$ photons $\mathrm{m}^{-2} \mathrm{~s}^{-1}$ ). Dotted horizontal lines indicate the mat surface. Open squares in panel (a) are the same data as those shown by solid squares, but plotted in a logarithmic scale. 
attenuation coefficient averaged over PAR was $\alpha=4.4 \mathrm{~mm}^{-1}$. The scalar irradiance directly under the surface $(0.1-0.2 \mathrm{~mm})$ was slightly enhanced in comparison to the scalar irradiance at the surface, due to light scattering and photon trapping in the mat (for example, see Kühl and Jørgensen, 1994; Kühl et al., 1996). Similar to previous observations (Jørgensen et al., 1987; Jørgensen and des Marais, 1988; Lassen et al., 1992b; Kühl and Fenchel, 2000), spectral light attenuation was strongly enhanced around wavelengths corresponding to the absorption maxima of predominant photopigments in the mat (Supplementary Figure S2a). Owing to this enhanced absorption, the irradiance reflectance, $R$, of the mat also exhibited pronounced minima around the same wavelengths (Supplementary Figure S2b).

When integrated over PAR, $17.5 \%$ of the incident irradiance was back-scattered from the microbial mat. The remaining $82.5 \%$ of the energy carried by the incident irradiance was thus absorbed in the mat, with $99 \%$ of this absorption taking place in the top $1 \mathrm{~mm}$. Chlorophyll $a$ autofluorescence constituted less than $0.03 \%, 0.024 \%$ and $0.16 \%$ of the incident blue, green and amber excitation, respectively, and could thus be neglected in the overall energy budget.

The thickness of the euphotic zone varied with the incident irradiance, increasing from $\sim 0.4 \mathrm{~mm}$ at $22 \mu \mathrm{mol}$ photon $\mathrm{m}^{-2} \mathrm{~s}^{-1}$ to $\sim 1.1 \mathrm{~mm}$ at $974 \mu \mathrm{mol}$ photon $\mathrm{m}^{-2} \mathrm{~s}^{-1}$ (Figure 1b). Besides a peak in PS found $0.3-0.4 \mathrm{~mm}$ below the mat surface at all irradiances, a second peak in photosynthetic activity emerged at a depth of $\sim 0.9 \mathrm{~mm}$ at incident irradiances $\geqslant 330 \mu$ mol photon $\mathrm{m}^{-2} \mathrm{~s}^{-1}$.

Areal rates of photosynthesis increased with the absorbed irradiance, $J_{\text {abs }}$, according to the saturated exponential model (Equation 5), reaching a maximum value of $P_{\max }=4.0 \mu \mathrm{mol} \mathrm{O}_{2} \mathrm{~m}^{-2} \mathrm{~s}^{-1}$ at $J_{\text {abs }}>800 \mu \mathrm{mol}$ photon $\mathrm{m}^{-2} \mathrm{~s}^{-1}$ (Figure 2a). Taking the initial slope of this photosynthesis vs absorbed irradiance relationship ( $\mathrm{P}-\mathrm{E}$ curve), the maximum $\mathrm{QE}$ of the microbial mat was $0.019 \pm 0.001 \mathrm{O}_{2}$ per photon. When converted to energy units, we obtained a relationship between the photosynthetically conserved energy and vector irradiance, that is, the net downwelling energy flux used here as a measure of the absorbed light energy (Figure 2a). The initial slope of this relationship, corresponding to the maximal energy conservation efficiency of the mat in light-limiting conditions, was $0.045 \pm 0.003$. The amount of photosynthetically conserved energy reached an asymptotic value of $\sim 1.9 \mathrm{~J} \mathrm{~m}^{-2} \mathrm{~s}^{-1}$ at vector irradiances above $\sim 200 \mathrm{~J} \mathrm{~m}^{-2} \mathrm{~s}^{-1}$.

The microbial mat temperature was only a fraction of a degree higher than in the overlying water (Figure 1c). However, the temperature difference increased with increasing irradiance, and a distinct thermal boundary layer was detectable at irradiances $>300 \mu \mathrm{mol}$ photon $\mathrm{m}^{-2} \mathrm{~s}^{-1}$ (vector irradiances $>54 \mathrm{~J} \mathrm{~m}^{-2} \mathrm{~s}^{-1}$ ). The flux of heat dissipated in the euphotic zone, as derived from the temperature gradient in thermal boundary layer (Equation 3), increased with the vector irradiance (Figure 2a). When using only data measured above $54 \mathrm{~J} \mathrm{~m}^{-2} \mathrm{~s}^{-1}$, that is, energy levels in which a temperature gradient in the thermal boundary layer was detectable, the slope of the linear fit was $0.969 \pm 0.035$.

A plot of the summed flux of energy conserved by photosynthesis and heat dissipation vs vector irradiance exhibited a linear relationship with a slope of $0.979 \pm 0.036$, which was close to the theoretically expected slope of 1 (Figure 2a). Assuming that the experimentally determined slope was exactly 1, we derived efficiencies of energy conservation and heat dissipation from Equation 4 and plotted them against the vector irradiance (Figure 2b). The plot shows that under light-limiting conditions $\sim 4.5 \%$ of the absorbed light energy was conserved by photosynthesis, whereas $\sim 95.5 \%$ was
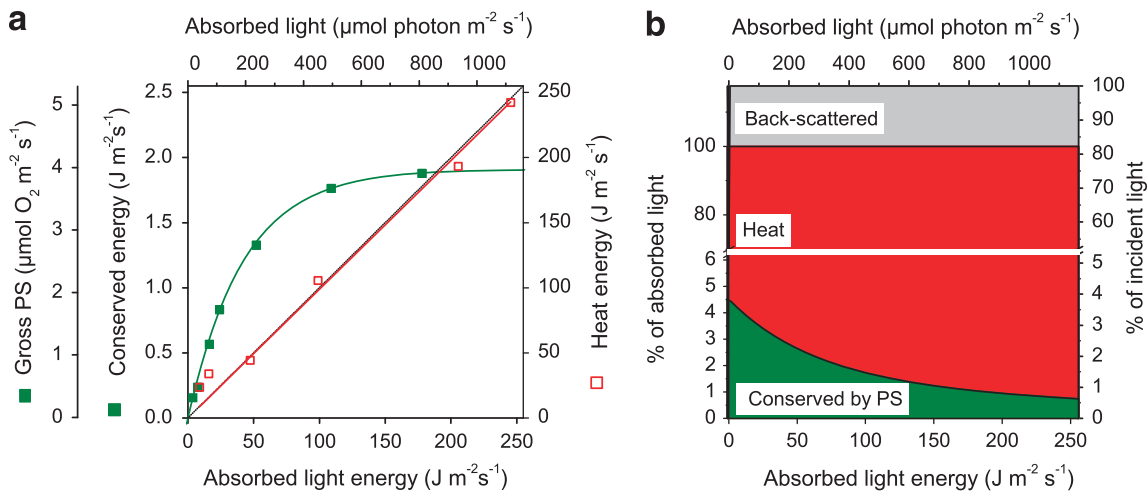

Figure 2 Energy budget in the studied mat. (a) Relationships between the areal rate of gross photosynthesis and vector irradiance (green symbols), and between the energy flux dissipated as heat and vector irradiance (red symbols), inside the mat. The vector irradiance, that is, the net downwelling light energy flux used here as a measure of the absorbed light, is expressed both in terms of quanta and energy. Green curve shows the fit by Equation $5\left(r^{2}=0.996\right)$, red line is the linear fit $\left(r^{2}=0.983\right)$ of used energy, that is, the sum of photosynthetically conserved energy and energy dissipated as heat, $J_{\mathrm{PS}}+J_{\mathrm{H}}$, vs absorbed light energy, $J_{\mathrm{abs}}$. For comparison, the theoretically expected relationship $J_{\mathrm{PS}}+J_{\mathrm{H}}=J_{\mathrm{abs}}$ is shown by the dotted line. (b) The relative disproportionation of the incident light energy among photosynthesis, heat and back-scattering at various levels of absorbed light energy in the mat. 
a

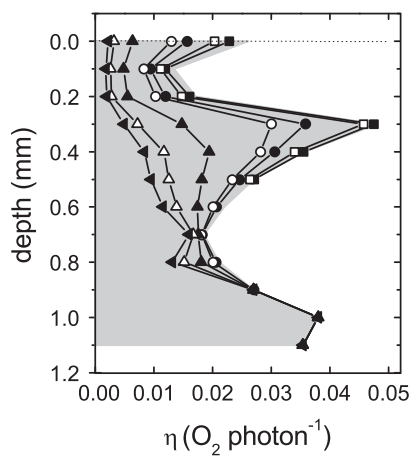

b

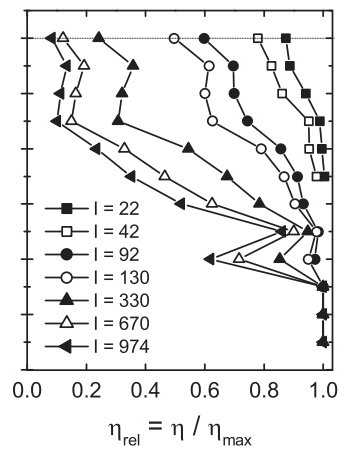

C

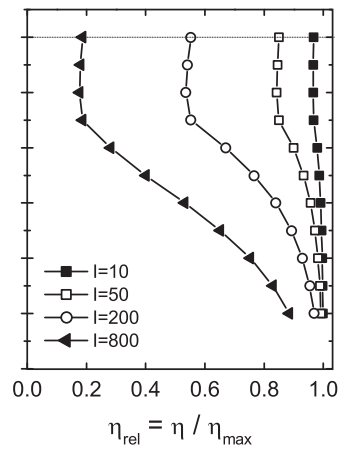

Figure 3 Vertical profiles of photosynthetic quantum efficiency (QE) in the studied microbial mat. (a) Local QE values, derived from data in Figures 1a,b, with the gray area indicating the maximum QE values in light-limiting conditions. (b) Relative local QE values. (c) Modeled depth profiles of relative local QE values, assuming that the maximum QE values and the maximum volumetric photosynthesis (PS) rates were independent of depth in the euphotic zone $\left(\eta_{\max }=0.025 \mathrm{O}_{2}\right.$ per photon, $\left.P_{\max }=4 \mu \mathrm{mol} \mathrm{O}_{2} \mathrm{~m}^{-3} \mathrm{~s}^{-1}\right)$. Legends indicate incident downwelling irradiance (integrated over photosynthetically active radiation (PAR)) in $\mu \mathrm{mol} \mathrm{photons} \mathrm{m}^{-2} \mathrm{~s}^{-1}$.

dissipated as heat. This energy disproportionation changed in favor of heat dissipation at increasing irradiance, with $>99 \%$ of the absorbed light energy being dissipated as heat and $<1 \%$ being used by photosynthesis at vector irradiances $>150 \mathrm{~J} \mathrm{~m}^{-2} \mathrm{~s}^{-1}$.

Throughout the euphotic zone, the increase in the volumetric rates of gross photosynthesis with the locally absorbed irradiance was generally fitted well $\left(r^{2}>0.88\right)$ by the saturated exponential model in Equation 5 (Supplementary Figure S3), which allowed calculation of the local QE values, $\eta$ (Equation 8). Maximum $\eta$ varied between 0.014-0.047 $\mathrm{O}_{2}$ per photon and exhibited two pronounced maxima at $0.3-0.4 \mathrm{~mm}$ and $1.0 \mathrm{~mm}$ below the mat surface (Figure 3a), coinciding with the measured maxima of gross photosynthesis rates (compare with Figure 1b). The local QE values gradually diminished with increasing irradiance, with the most pronounced decrease observed in the upper $\sim 0.5 \mathrm{~mm}$ of the mat. This effect is better illustrated in Figure $3 \mathrm{~b}$; for example, although the local QE values at depths $0-0.5 \mathrm{~mm}$ decreased to $10-50 \%$ of the maximum efficiency at incident quantum irradiances $>300 \mu \mathrm{mol}$ photon $\mathrm{m}^{-2} \mathrm{~s}^{-1}$, the local QE values in the deeper parts of the mat remained around $70-100 \%$ of the maximum values.

\section{Discussion}

At the cellular level, optimal light utilization in oxygenic phototrophs follows the well-established Z-scheme, whereby energy of the photons absorbed by the light harvesting complex drives a series of redox reactions after an initial charge separation across the photosynthetic membrane, resulting in the generation of a proton gradient (used for ATP synthesis) and reduction equivalents. By coupling to the $\mathrm{CO}_{2}$ fixation machinery and nutrient uptake, this process eventually results in the conversion of solar energy to chemical energy bound in organic-C compounds. It also leads to evolution of molecular oxygen as a byproduct. At very low photon fluxes, the rate of photosynthesis is limited by the rate of excitation energy supply to the photosystems, the efficiency of primary photochemistry and the stoichiometry of the two photoreactions required to generate the appropriate ATP and NADPH stoichiometry for $\mathrm{O}_{2}$ evolution and $\mathrm{CO}_{2}$ fixation. At higher photon fluxes, the redox reactions limit the rate of biochemical energy transformation, and only part of the absorbed light energy is actually stored, whereas the rest is dissipated non-photochemically as heat and fluorescence. Our measurements show that such a conceptual model of energy flow can be formulated and experimentally validated for a photosynthetic microbial mat, which represents a complex microbial ecosystem characterized by a high density of phototrophic (and other) cells and steep light gradients.

We demonstrated that the sum of photosynthetically stored energy, $J_{\mathrm{PS}}$, and energy dissipated as heat, $J_{\mathrm{H}}$, accounted well for the total energy absorbed by the microbial mat, $J_{\text {abs }}$. At high irradiance, the discrepancy between $J_{\mathrm{PS}}+J_{\mathrm{H}}$ and $J_{\mathrm{abs}}$ was only about $2 \%$. This was considered satisfactory to prove our original hypothesis about the energy flow, especially when taking into account the experimental challenges involved, such as the necessity to conduct accurate light, temperature and photosynthesis measurements at sub-millimeter spatial resolution. At low irradiance, the discrepancy in the energy balance was larger; for example, although the slope of $J_{\mathrm{H}}$ vs $J_{\text {abs }}$ was expected to decrease at low $J_{\text {abs }}$ (due to proportionally higher contribution of photosynthesis to light energy utilization), this was not clear from our data, mainly because the temperature gradients measured at $J_{\mathrm{abs}}<50 \mathrm{~J} \mathrm{~m}^{-2} \mathrm{~s}^{-1}$ were close to or below the detection limit of the temperature microsensor. Therefore, the determination of the energy conversion efficiencies at low irradiance relied on the measurements of photosynthesis 
and on the assumption that the energy balance $J_{\mathrm{abs}}=J_{\mathrm{PS}}+J_{\mathrm{H}}$ remained valid for the entire range of investigated irradiances, although it could be experimentally verified with satisfactory accuracy only at high irradiances.

In the studied microbial mat, a relatively large portion $(17.5 \%)$ of the incident photosynthetically active radiation was back-scattered and thus not used by the mat. Auto-fluorescence emitted by pigments in the mat constituted only a negligible fraction of the total energy budget. The remaining $82.5 \%$ of the incident irradiance was absorbed within the top $0.4-1.1 \mathrm{~mm}$ of the mat. The majority of absorbed energy was dissipated as heat, whereas chemically conserved energy constituted less than $4.5 \%$ (Figure 2b). The highest energy storage efficiency of the mat occurred under light-limiting conditions. With increasing irradiance, the photosynthetic efficiency decreased approximately inversely with the absorbed light energy ( $\propto J_{\text {abs }}^{-1}$ ), reaching less than $1 \%$ at $J_{\mathrm{abs}}>300 \mathrm{~J} \mathrm{~m}^{-2} \mathrm{~s}^{-1}$.

At the cellular level, the fact that photosynthesis follows a saturation curve, and that its efficiency therefore decreases, is foremost due to the enzymatic processes in the photosynthetic apparatus becoming rate limiting at increasing irradiance. Additional factors contributing to saturation, or even to inhibition at high irradiances, include enhanced photorespiration or cyclic photophosphorylation, which generate some ATP, or the formation of reactive oxygen species that can affect photosystem II by damaging the D1 protein or by preventing its repair (Hihara et al., 2001; Nishiyama et al., 2001, 2004; Aarti et al., 2007; Latifi et al., 2009). Overall, the saturation dynamics of photosynthesis with irradiance (P-E curve) can be described by a number of empirical models (for example, Equation 5; Webb et al., 1974; also see MacIntyre et al., 2002). As demonstrated by the P-E curves shown in Supplementary Figure S3, a qualitatively similar physiological mechanism regulates photosynthetic activity in each individual layer of the mat's euphotic zone. However, although one can expect that the changes in the photosynthetic efficiency on the ecosystem and cellular level are linked, the relationship between the two is complicated by the fact that the light present in the ecosystem exhibits a pronounced gradient (for example, the upper-most part of the euphotic zone experienced $\sim 100$-fold higher light levels than the lower-most one; Figure 1a).

This relationship can be qualitatively understood by examining the vertical distribution of the local photosynthetic efficiencies as a function of irradiance. Comparing Figures 2a and 3a, b, it is clear that the energy storage efficiency of the entire mat in light-limiting conditions is maximal because all layers within the euphotic zone use the locally available light with maximum efficiency. This implies that the areal photosynthesis rate should initially increase approximately linearly with incident irradiance, which was indeed observed. An increase in the incident irradiance leads to an increase in local light availability for progressively deeper mat regions, with the upper layers experiencing a much higher increase compared with the deeper mat layers because of a steep light gradient in the mat. Consequently, the upper mat layers experience a more severe decrease in photosynthetic efficiency than the lower ones. The overall effect is a decrease in efficiency of the entire mat. However, the resulting value lies between those exhibited by the upper and lower parts of the euphotic zone, because it is a weighted average over all photosynthetically active layers. Such a decrease in efficiency results in the observed departure of the P-E relationship (areal rates) from the straight line towards a curve with a lower derivative (Figure 2a). This trend continues with increasing irradiance until the efficiency in most of the euphotic zone is very low (Figures $3 a$ and $b$, triangles), leading to the observed saturation in the areal rate of gross photosynthesis (Figure 2a). We confirmed this conceptual description by a simplified model, assuming similar but constant values for the maximum QE and the maximum volumetric rate of photosynthesis as those determined experimentally throughout the entire euphotic zone. By using the measured light attenuation in the euphotic zone in the model, we were able to reproduce the observed spatial heterogeneities in the decrease of the relative photosynthetic efficiency with the increasing irradiance levels (compare Figures $3 \mathrm{~b}$ and $\mathrm{c}$ ).

The maximum QE $\left(0.019 \mathrm{O}_{2}\right.$ per photon) in the studied mat is about sixfold lower than the theoretical maximum, and is also at the lower end of values compiled from studies of various photosynthetic organisms, including higher plants, corals, macro- and microalgae (Supplementary Table S1). It needs to be realized, however, that our value represents the overall efficiency of the entire mat ecosystem and not of the actual phototrophic cells. In mats, a large proportion of the light energy is likely absorbed by abiotic (for example, sediment particles or $\mathrm{Fe}$ precipitates) or biotic but photosynthetically inactive components (for example, protective sunscreen pigments, detritus or phaeopigments). Thus, the true photosynthetic efficiency of the phototrophic cells comprizing the mat is likely higher than estimated from our measurements. Furthermore, there are other processes on the cellular level that cause uncoupling between light utilization, $\mathrm{O}_{2}$ production and $\mathrm{CO}_{2}$ fixation, and thus effect the estimated QE. For example, in cyclic photophosphorylation the energy of absorbed photons is stored as ATP but no $\mathrm{O}_{2}$ is evolved, resulting in an underestimated QE based on $\mathrm{O}_{2}$ measurements. The Mehler reaction has a similar qualitative effect on QE, because $\mathrm{O}_{2}$ generated from oxidation of water is reduced to $\mathrm{H}_{2} \mathrm{O}_{2}$ and ultimately back to water, with some ATP production. However, photorespiration can result in an overestimated QE 
values when determined from $\mathrm{O}_{2}$ measurements, because ribulose-1,5-bisphosphate carboxylase/oxygenase reacts competitively with $\mathrm{O}_{2}$ instead of $\mathrm{CO}_{2}$, which leads to lower $\mathrm{CO}_{2}$ fixation than the measured $\mathrm{O}_{2}$ evolution. Furthermore, part of the electrons generated from water oxidation are used for other reductive processes in the cell, such as reduction of nitrogen and sulfur, leading also to QE overestimation. Although the effects of these additonal processes are expected not to exceed $10 \%$ of the estimated QE at light-limiting conditions (Falkowski and Raven, 1997), they are probably more significant under higher photon fluxes, especially because of a strong build-up of $\mathrm{O}_{2}$, reactive oxygen species and $\mathrm{pH}$ in the euphotic zone. However, an accurate quantification of such effects was not possible in our experimental approach.

The maximum $\mathrm{QE}$ in the studied mat exhibited a pronounced vertical stratification (Figure 3a). The peaks at depths of $0.3-0.4$ and $1 \mathrm{~mm}$ could be either due to the phototrophic cells being proportionally more abundant in these layers than the photosynthetically inactive mat components, or because the phototrophic cells were indeed physiologically adapted or acclimated to capture and use photons more efficiently (for example, by having a higher pigment content and/or absoption cross section). The position of these layers was probably the result of cell growth at optimal light conditions within the gradient light field generated by the incident light during mat growth. A detailed clarification of these interesting aspects would go beyond the scope of this study, but could be experimentally investigated, for example, by conducting microscale variable fluorescence measurements to map photosynthetic biomass in concert with quantum yields (Schreiber et al., 1996; Kühl, 2005), or by a range of new imaging methods (Kühl and Polerecky, 2008).

The overall EE of the studied mat ecosystem was in the order of $1-2 \%$ at irradiances corresponding to typical daytime values $(100-1000 \mu$ mol photon $\mathrm{m}^{-2} \mathrm{~s}^{-1}$ ). This EE value may seem low when compared with the theoretical maximum of $27.7 \%$ or with the maximum efficiencies of individual organisms, such as plants or microalgae (Supplementary Table S1). However, the studied microbial mat is about 10-20 times more efficient than the estimated primary productivity of the global ecosystem, which converts around $0.1 \%$ of the available light to biomass (Makarieva et al., 2008).

Besides presenting a new experimental approach for studying photosynthetic and energy efficiencies, this study also presents a theoretical framework for the description of light energy propagation, conversion and conservation in benthic photosynthetic systems such as microbial mats (see Supplementary Information). Owing to a high density of pigmented cells and other particles, light is intensively scattered and absorbed as it propagates through the mat. This results in a rapid switch from incident predominantly collimated light to diffuse light that is highly attenuated with depth in the mat (Kühl and Jørgensen, 1992). Previous study showed that light absorption at a specific depth in the mat could be quantified from combined measurements of the downwelling and upwelling irradiance and the scalar irradiance (Kühl and Jørgensen, 1994). However, in practice such measurements are laborious and difficult to use in complex samples.

In this study, we show that the locally absorbed light can be estimated from the locally available light. The proposed calculation (see Supplementary Information) was adapted from the work of Yang et al. (2004), who extended the original KubelkaMunk theory of light propagation in absorbing and scattering media. The essential step was the realization that (i) the downwelling and upwelling photon fluxes, for which the Kubelka-Munk formalism was originally developed, are proportional to the scalar irradiance if a totally diffused light field is assumed, and that (ii) the absorption coefficient required to calculate the absorbed light can be derived from the light attenuation coefficient obtained from the vertical profile of scalar irradiance and the reflectance of the mat (see Equation 7 and Supplementary Information). As all of these parameters can easily be measured, this is a major advantage which now makes it possible to calculate photosynthetic efficiencies inside dense assemblages of cells such as benthic biofilms or mats (Equation 8). However, one needs to be cautious with interpretation within the top $0.1-0.2 \mathrm{~mm}$ of the mat, where the light field is anisotropic (Kühl and Jørgensen, 1994). However, this region forms only a relatively small part of the euphotic zone.

In conclusion, this study presents, to the best of our knowledge, the first balanced assessment of the fate of light energy inside a microphytobenthic ecosystem. The energy budget is dominated by heat dissipation and the efficiency of photosynthetic energy conservation decreases with the increasing irradiance. Owing to steep light gradients, this decrease is highly heterogeneous across the euphotic zone. Moreover, we derived a mathematical formula to quantify the locally absorbed light from easily measured parameters, that is, the light attenuation coefficient, the reflectance and the scalar irradiance. On the basis of this study, it is now possible to address the photobiology of densely populated biofilms and microbial mats with intense absorption and scattering in much more detail, but our analysis may also find application in other areas of photosynthesis research, such as plant biology and biotechnology, for example, while optimizing quantum yields and growth efficiency of phototrophic cell cultures for biofuel production.

\section{Acknowledgements}

We thank the technicians of the microsensor group for microsensor construction and Paul Faerber, Harald 
Osmers, Georg Herz and Volker Meyer for their technical support. We thank Dr Henk Jonkers and Prof Friedrich Widdel for fruitful discussions, and Prof Waleed Hamza for his guidance and assistance during sampling. Two anonymous reviewers are specially thanked for their useful comments and suggestions to improve the paper. This study was financially supported by the Max Planck Society (to DB, BBJ and LP), the Yusef Jameel foundation (to MA-N) and the Danish Natural Science Research Council (to MK).

\section{References}

Aarti D, Tanaka R, Ito H, Tanaka A. (2007). High light inhibits chlorophyll biosynthesis at the level of 5aminolevulinate synthesis during de-etiolation in cucumber (Cucumis sativus) cotyledons. Photochem Photobiol 83: 171-176.

Cahoon LB. (1999). The role of benthic microalgae in neritic ecosystems. Oceanogr Mar Biol 37: 47-86.

Dismukes GC, Carrieri D, Bennette N, Ananyev GM, Posewitz MC. (2008). Aquatic phototrophs: efficient alternatives to land-based crops for biofuels. Curr Opin Biotechnol 19: 235-240.

Dubinsky Z, Falkowski PG, Wyman K. (1986). Light harvesting and utilization by phytoplankton. Plant Cell Physiol 27: 1335-1349.

Falkowski PG, Raven JA. (1997). Aquatic photosynthesis. Blackwell Science. Capital City Press: Washington, DC.

Flameling IA, Kromkamp J. (1998). Light dependence of quantum yields for PSII charge separation and oxygen evolution in eucaryotic algae. Limnol Oceanogr 43: 284-297.

Guerrero R, Piqueras M, Berlanga M. (2002). Microbial mats and the search for minimal ecosystems. Int Microbiol 5: 177-188.

Hihara Y, Kamei A, Kanehisa M, Kaplan A, Ikeuchi M. (2001). DNA microarray analysis of cyanobacterial gene expression during acclimation to high light. Plant Cell 13: 793-806.

Huner NPA, Oquist G, Sarhan F. (1998). Energy balance and acclimation to light and cold. Trends Plant Sci 3: 224-230.

Jimenez IM, Kühl M, Larkum AWD, Ralph PJ. (2008). Heat budget and thermal microenvironment of shallowwater corals: do massive corals get warmer than branching corals? Limnol Oceanogr 53: 1548-1561.

Jørgensen BB, Cohen Y, des Marais DJ. (1987). Photosynthetic action spectra and adaptation to spectral light-distribution in a benthic cyanobacterial mat. Appl Environmental Microbiol 53: 879-886.

Jørgensen BB, des Marais DJ. (1988). Optical-properties of benthic photosynthetic communities-fiber-optic studies of cyanobacterial mats. Limnol Oceanogr 33: 99-113.

Kühl M. (2005). Optical microsensors for analysis of microbial communities. Methods Enzymol 397: 166-199.

Kühl M, Fenchel T. (2000). Bio-optical characteristics and the vertical distribution of photosynthetic pigments and photosynthesis in an artificial cyanobacterial mat. Microb Ecol 40: 94-103.

Kühl M, Glud RN, Ploug H, Ramsing NB. (1996). Microenvironmental control of photosynthesis and photosynthesis-coupled respiration in an epilithic cyanobacterial biofilm. J Phycol 32: 799-812.

Kühl M, Jørgensen BB. (1992). Spectral light measurements in microbenthic phototrophic communities with a fiber-optic microprobe coupled to a sensitive diode array detector. Limnol Oceanogr 37: 1813-1823.

Kühl M, Jørgensen BB. (1994). The light-field of microbenthic communities-radiance distribution and microscale optics of sandy coastal sediments. Limnol Oceanogr 39: 1368-1398.

Kühl M, Lassen C, Jørgensen BB. (1994). Light penetration and light intensity in sandy marine sediments measured with irradiance and scalar irradiance fiber-optic microprobes. Mar Ecol Prog Ser 105: 139-148.

Kühl M, Polerecky L. (2008). Functional and structural imaging of phototrophic microbial communities and symbioses. Aquat Microb Ecol 53: 99-118.

Lassen C, Ploug H, Jørgensen BB. (1992a). A fiberoptic scalar irradiance microsensor-application for spectral light measurements in sediments. FEMS Microbiol Ecol 86: 247-254.

Lassen C, Ploug H, Jørgensen BB. (1992b). Microalgal photosynthesis and spectral scalar irradiance in coastal marine-sediments of Limfjorden, Denmark. Limnol Oceanogr 37: 760-772.

Latifi A, Ruiz M, Zhang CC. (2009). Oxidative stress in cyanobacteria. FEMS Microb Rev 33: 258-278.

MacIntyre HL, Kana TM, Anning T, Geider RJ. (2002). Photoacclimation of photosynthesis irradiance response curves and photosynthetic pigments in microalgae and cyanobacteria. J Phycol 38: 17-38.

Makarieva AM, Gorshkov VG, Li BL. (2008). Energy budget of the biosphere and civilization: rethinking environmental security of global renewable and nonrenewable resources. Ecol Complex 5: 281-288.

Mussgnug JH, Thomas-Hall S, Rupprecht J, Foo A, Klassen V, McDowall A et al. (2007). Engineering photosynthetic light capture: impacts on improved solar energy to biomass conversion. Plant Biotechnol J 5: $802-814$.

Nishiyama Y, Allakhverdiev SI, Yamamoto H, Hayashi H, Murata N. (2004). Singlet oxygen inhibits the repair of photosystem II by suppressing the translation elongation of the D1 protein in Synechocystis sp PCC 6803. Biochemistry 43: 11321-11330.

Nishiyama Y, Yamamoto H, Allakhverdiev SI, Inaba M, Yokota A, Murata N. (2001). Oxidative stress inhibits the repair of photodamage to the photosynthetic machinery. EMBO J 20: 5587-5594.

Osmond CB. (1994). What is photoinhibition? Some insights from comparisons of shade and sun plants. In: NR Baker and JR Bowyer (eds). Environmental Plant Biology Series; Photoinhibition of photosynthesis: From molecular mechanisms to the field. Bios Scientific Publisher: Oxford. pp 1-24.

Ragauskas AJ, Williams CK, Davison BH, Britovsek G, Cairney J, Eckert CA et al. (2006). The path forward for biofuels and biomaterials. Science 311: 484-489.

Revsbech NP. (1989). An oxygen microsensor with a guard cathode. Limnol Oceanogr 34: 474-478.

Revsbech NP, Jørgensen BB. (1983). Photosynthesis of benthic microflora measured with high spatial-resolution by the oxygen microprofile method-capabilities and limitations of the method. Limnol Oceanogr 28: 749-756.

Revsbech NP, Jørgensen BB. (1986). MicroelectrodesTheir use in microbial ecology. Adv Microb Ecol 9: 293-352. 
Revsbech NP, Jørgensen BB, Blackburn TH, Cohen Y. (1983). Microelectrode studies of the photosynthesis and $\mathrm{O}_{2}, \mathrm{H}_{2} \mathrm{~S}$, and $\mathrm{pH}$ profiles of a microbial mat. Limnol Oceanogr 28: 1062-1074.

Rosenberg JN, Oyler GA, Wilkinson L, Betenbaugh MJ. (2008). A green light for engineered algae: redirecting metabolism to fuel a biotechnology revolution. Curr Opin Biotechnol 19: 430-436.

Schneider TR. (1973). Efficiency of photosynthesis as a solar energy converter. Energy Conversion 13: 77-84.

Schreiber U, Kühl M, Klimant I, Reising H. (1996). Measurement of chlorophyll fluorescence within leaves using a modified PAM Fluorometer with a fiber-optic microprobe. Photosynth Res 47: 103-109.

Singsaas EL, Ort DR, DeLucia EH. (2001). Variation in measured values of photosynthetic quantum yield in ecophysiological studies. Oecologia 128: 15-23.
Stal LJ. (2000). Cyanobacterial mats and stromatolites. In: $\mathrm{AB}$ Whitton and $\mathrm{M}$ Potts (eds). The Ecology of Cyanobacteria. Kluwer Academic Publishers: Dordrecht. pp 61-120.

Thauer RK, Jungermann K, Decker K. (1977). Energy conservation in chemotrophic anaerobic bacteria. Bacteriol Rev 41: 100-180.

vanGemerden H. (1993). Microbial mats: a joint venture. Mar Geol 113: 3-25.

Webb WL, Newton M, Starr D. (1974). Carbon-dioxide exchange of Alnus-Rubra-mathematical model. Oecologia 17: 281-291.

Yang L, Kruse B, Miklavcic SJ. (2004). Revised KubelkaMunk theory. II. Unified framework for homogeneous and inhomogeneous optical media. J Opt Soc Am A Opt Image Sci Vis 21: 1942-1952.

Zhu XG, Long SP, Ort DR. (2008). What is the maximum efficiency with which photosynthesis can convert solar energy into biomass? Curr Opin Biotechnol 19: 153-159.

Supplementary Information accompanies the paper on The ISME Journal website (http://www.nature.com/ismej) 